\title{
Desafios institucionais à inclusão de estudantes com Necessidades Educativas Especiais no Ensino Superior
}

\author{
Maria Leonor Borgesi \& Maria Helena Martins ii $^{\mathrm{i}}$ \\ Universidade do Algarve, Portugal \\ Emilio Lucio-Villegasiii \\ Universidad de Sevilla, España
}

Teresa Gonçalvesiv

Universidade do Algarve, Portugal

Resumo

Com base nos pressupostos da educação inclusiva e crescente democratização do ensino superior temos vindo a verificar a progressiva abertura da universidade a diversas minorias, nomeadamente estudantes com Necessidades Educativas Especiais (NEE). Constata-se que ainda há pouco conhecimento sobre o tema da diversidade e inclusão entre docentes e estudantes, em geral, sendo que os serviços institucionais de apoio aos estudantes com NEE existentes carecem de eficácia em diversos níveis, nomeadamente ao nível das atitudes dos docentes e pessoal não-docente. $\mathrm{O}$ que pensam estes atores institucionais sobre a inclusão destes estudantes? Proporcionam as universidades as condições adequadas a esta minoria estudantil? Através de um estudo de caso na Universidade do Algarve, pretendeu-se analisar as conceções e atitudes dos docentes e pessoal nãodocente face à inclusão dos estudantes com NEE no Ensino Superior. Pretendeu-se ainda conhecer quais os desafios que estes atores institucionais assinalam no âmbito da inclusão. Tendo por referência os seus testemunhos, constata-se que, apesar do esforço da instituição em análise, continuam a existir áreas que necessitam de um maior investimento, desde a remoção das 
barreiras arquitetónicas, ao desenvolvimento de atitudes mais inclusivas da comunidade académica, passando por práticas de ensino-aprendizagem e de avaliação, que assegurem a igualdade e equidade.

\section{Palavras-chave}

Educação Inclusiva; Necessidades Educativas Especiais; Ensino Superior

\section{Introdução}

Nas últimas décadas a deficiência tem vindo a ser conceptualizada como uma questão de direitos humanos (UNESCO, 2017; United Nations, 2015). Não obstante, o relatório Education and Disability/Special Needs, Policies and Practices in Education, Training and Employment for Students with Disabilities and Special Educational Needs in the EU (European Commission, 2012) revela que mais de 15 milhões de crianças e jovens com Necessidades Educativas Especiais (NEE) continuam sem acesso às oportunidades de educação e de emprego.

As implicações da desigualdade de oportunidades em diversos contextos, como na inserção no mercado de trabalho, no desenvolvimento da vida profissional e pessoal, no nível de rendimentos, no acesso à saúde, à habitação e no pleno desenvolvimento da cidadania, exigem a garantia no acesso das pessoas com NEE à educação (Capucha, 2010; UNESCO, 2017).

Em particular, a inclusão dos estudantes com NEE no Ensino Superior (ES) não tem sido consensual e a participação e sucesso académico dos mesmos encontram diversos constrangimentos que urge analisar e a que é necessário responder de forma a guiar a mudança institucional e levar à assunção das responsabilidades que o ES tem para com o desenvolvimento social (Abreu, 2011; Antunes \& Faria, 2013; Berggren, Rowan, Bergbäck, \& Blomberg, 2016; Bisol \& Valentin, 2012; Cabral, Mendes, de Anna, \& Ebersold, 2015; Foundation of Tertiary Institutions of the Northern Metropolis [FOTIM], 2011; Fragoso et al., 2015). A investigação desenvolvida por França (2014) partilha da mesma opinião, assinalando que, dependendo do tipo de NEE que o estudante apresenta, ao ingressar na universidade encontra diversas barreiras atitudinais que dificultam o seu processo de ensino-aprendizagem. 
A análise da literatura revela diversos estudos realizados em Portugal sobre a inclusão no ES, focalizando fundamentalmente as perceções dos estudantes com NEE (Abreu, 2011; Antunes \& Faria, 2013; Castanheira, 2013; Curado \& Oliveira, 2010; Fernandes \& Almeida, 2007; Gonçalves \& Cardoso, 2010; Porfírio, Gronita, Carrilho, \& Silva, 2016; Santos, Gonçalves, Ramos, Castro, \& Lomeo, 2015).

\section{A inclusão no Ensino Superior: Do panorama internacional ao caso português}

A inclusão, enquanto movimento social e político, é sinónima de luta contra todas as formas de desigualdade social que afetam as minorias (Runswick-Cole, 2011). Runswick-Cole (2011) assinala ainda que a inclusão defende o direito de todos os indivíduos participarem, de uma forma consciente e responsável, na sociedade, de serem aceites e respeitados, independentemente do que os diferencia. Isto significa que a inclusão pretende alcançar o objetivo de caminhar para a equidade de todos, reconhecendo e apoiando a riqueza da diversidade social e contrariando, no caso dos indivíduos com NEE, os redutores parâmetros culturais de normalidade (Runswick-Cole, 2011). Enquanto movimento educacional, a inclusão centra-se na necessidade de as escolas mudarem as suas culturas e práticas de forma a garantir uma educação adequada a todas as pessoas, promovendo a sua participação na sociedade, escola ou comunidade, diminuindo e eliminando todo o tipo de processos que levam à exclusão social. Neste âmbito, poderíamos questionar se a mensagem que se transmite em muitas ocasiões é a mensagem de que o diferente deve ser escondido dos espaços públicos, por ser considerado irrelevante ou invisível (Berggren et al., 2016; Campbell, Fontes, Hemingway, Soorenian, \& Till, 2008). Esta visão cria uma categorização social que parece estar presente no ES e, nesse sentido, seria importante explorar modelos sociais que forneçam ferramentas analíticas tanto para análise académica da deficiência como para a ação política" (Campbell et al., 2008, p. 31). Urge abandonar um modelo social de deficiência que se centra no que está errado em cada um de nós e que se questione, antes, sobre o que está errado a nível institucional. Esta posição significa assumir que as instituições de Ensino Superior (IES) são libertadoras, e não instituições que procuram uma classificação social 
baseada em variáveis como o género, a etnia, ou, neste contexto particular, as necessidades educativas especiais (Runswick-Cole, 2011).

As questões da educação inclusiva e do acesso à universidade para as pessoas com NEE constituem, assim, uma temática extremamente relevante, quer no âmbito nacional, quer internacional (Bisol \& Valentin, 2012; Harrison, Hemingway, Sheldon, Pawson, \& Barnes, 2009; Santos et al., 2015; UNESCO, 2017). Efetivamente, tendo em consideração as orientações e políticas mundiais, cada país tem vindo a definir as suas prioridades, estratégias e legislação de suporte aos direitos dos Estudantes com NEE (ENEE) (Melo \& Martins, 2016). Importa, contudo, assinalar que a presença destes estudantes no ES continua a ser minoritária, marcada por abandono precoce e insucesso académico (Gonçalves \& Cardoso, 2010; Moreira, Bolsanello, \& Seger, 2011; Nolan, Gleeson, Treanor, \& Madigan 2015), apontando-se como causas fundamentais as barreiras arquitetónicas, a falta de adequação dos processos de ensino-aprendizagem, a desadequação do processo e/ou instrumentos de avaliação, a ausência de recursos educativos adequados e, principalmente, as barreiras atitudinais (European Commission, 2012; FOTIM, 2011; UNESCO, 2017).

Em Portugal verifica-se que se para o ensino básico e secundário a legislação e as práticas têm sido mais consistentes, no ES escasseia a legislação (Bisol \& Valentin, 2012; Castanheira, 2013; Fernandes \& Almeida, 2007; Melo \& Martins, 2016; Santos et al., 2015). Na lei que define as "Bases Gerais do Regime Jurídico da Prevenção, Habilitação, Reabilitação e Participação da Pessoa com Deficiência" (Lei n. ${ }^{\circ}$ 38/2004) é acometida ao Estado português a responsabilidade da "execução de uma política de prevenção, habilitação, reabilitação e participação da pessoa com deficiência". No entanto, a Lei de Bases do Sistema Educativo (Lei n. ${ }^{\circ}$ 46/86) apenas reconhece de forma expressa apoio específico nos estabelecimentos de ensino básico e secundário. Com exceção feita à legislação que regulamenta o acesso ao ES e ao normativo sobre o contingente especial para candidatos com deficiência física ou sensorial, não existem na legislação atual diretrizes que garantam equidade aos ENEE durante o seu percurso académico universitário (Fragoso et al., 2015; Melo \& Martins, 2016).

As universidades e institutos politécnicos, confrontados, por um lado, com o aumento do número de ENEE, e, por outro, com as orientações 
políticas internacionais e nacionais em prol da educação inclusiva, têm procurado implementar internamente medidas de apoio adequadas às necessidades deste público (Grupo de Trabalho para o Apoio a Estudantes com Deficiência no Ensino Superior [GTAEDES], 2014). Efetivamente, este aumento de estudantes no ES produziu uma maior conscientização, no seio da comunidade académica, acerca da necessidade de adaptação a esta realidade, gerando respostas institucionais diversas (Couzens et al., 2015; Nolan et al., 2015; Venville, Street, \& Fossey, 2014). As perspetivas que orientam o apoio aos ENEE têm sido diversas nas últimas décadas, influenciando o desenvolvimento de políticas e provisões (Berggren et al., 2016). De assinalar, em Portugal, a criação de um Grupo de Trabalho para o Apoio a Estudantes com Deficiência no Ensino Superior - GTAEDES, que é constituído por representantes de diversas universidades portuguesas e que tem como principais objetivos a partilha de experiências, a divulgação de informações e a definição de normas orientadoras de boas práticas de inclusão no ES (GTAEDES, 2014).

Outra das respostas da comunidade académica tem sido a criação de serviços ou gabinetes de apoio nas universidades. Estes serviços oferecem apoios específicos especializados aos ENEE mas também aos docentes e pessoal não-docente, que, na maior parte das vezes, referem não ter formação específica para trabalhar com estes estudantes (Gonçalves, Borges, \& Martins, 2014; Martins, Borges, Gonçalves, \& Fonseca, 2014). Efetivamente, diversas investigações assinalam o papel fundamental que o docente do ES desempenha para que a inclusão possa ser uma realidade (Abu-Hamour, 2013; Antunes, Faria, Rodrigues, \& Almeida, 2013; Bisol \& Valentin, 2012; Cabral et al. 2015; Couzens et al., 2015; Fossey et al., 2017; French et al., 2014; Mamah, Deku, Darling, \& Avoke, 2011; Meira \& Pereira, 2017; Morgado, Melero, Molina, \& Cortés-Vega, 2016; Rodrigues, 2015).

Tendo em atenção a importância das atitudes e acessibilidades para a promoção da inclusão no ES (Faria, 2012; França, 2014; Mamah et al., 2011; Melo \& Martins, 2016), aliada à escassez de estudos sobre as perceções dos professores acerca da inclusão no ES em Portugal (Bisol \& Valentim, 2012; Faria, 2012; Melo \& Martins, 2016), parece relevante investigar e conhecer as atitudes, as conceções que enquadram as práticas na universidade, a partir dos relatos dos seus atores institucionais, numa instituição de ES em 
Portugal. Assim, através de um estudo de caso na Universidade do Algarve, pretendeu-se analisar as conceções e atitudes dos docentes e pessoal nãodocente face à inclusão dos ENEE no ES. Pretendeu-se ainda conhecer quais os desafios que estes atores institucionais assinalam no âmbito da inclusão.

\section{Contexto e metodologia}

Os resultados que se apresentam integram a investigação "Estudantes não-tradicionais no Ensino Superior: Investigar para guiar a mudança institucional"1, que envolveu as Universidades do Algarve e de Aveiro, apresentando-se, no presente artigo, apenas alguns resultados parciais. Nesta investigação pretendeu-se analisar o processo de inclusão de estudantes não-tradicionais - estudantes oriundos dos PALOP, estudantes maiores de 23 anos, estudantes dos Cursos de Especialização Tecnológica e ENEE - com vista à elaboração de recomendações que possam orientar a mudança institucional e levem à assunção das responsabilidades que o ES tem para com o desenvolvimento social. O estudo estruturou-se através de um paradigma de investigação interpretativa de cariz qualitativo, privilegiandose o recurso a entrevistas semiestruturadas, realizadas a estudantes com NEE, a professores e diretores de curso destes alunos, elementos da gestão académica e pessoal técnico. Foi ainda realizado um focus group com os colegas dos estudantes com NEE.

Atendendo à importância das atitudes e acessibilidades para a promoção da inclusão no ES, optou-se por apresentar, no presente artigo, as conceções e atitudes dos docentes, diretores de curso, diretores de Unidades Orgânicas e pessoal não-docente face à inclusão dos ENEE na Universidade do Algarve (UAlg).

Após análise da literatura da área e de diversos estudos realizados, foram construídos os guiões das entrevistas, que incidiram sobre diversos temas, como: a inclusão em instituições de ES; as atitudes em relação à educação inclusiva; o apoio pedagógico; o processo de ensino-aprendizagem; e os obstáculos identificados. Depois de gravadas em áudio e transcritas as entrevistas, o corpus da informação foi objeto de análise de conteúdo temática, tendo-se utilizado um sistema de codificação categorial sugerido por Bardin (2009). A amostragem é não-probabilística, de conveniência (Patton, 
1990), tendo sido selecionada a partir de população acessível e disponível para participar no estudo.

Os resultados que se apresentam reportam-se a uma amostra constituída por um total de 18 participantes afetos às diversas Unidades Orgânicas e serviços da Universidade do Algarve. Como critério de inclusão, os inquiridos deveriam ter tido já contacto e/ou lecionado em turmas em que estavam incluídos ENEE2 ${ }^{2}$. Houve a preocupação de entrevistar, em cada uma das Unidades Orgânicas, os diretores, os docentes e os diretores dos cursos. Também os técnicos de Ação Social e a bibliotecária que participaram já tinham tido diversos contactos com ENEE. A amostra incluiu seis docentes $\left(\mathrm{P}^{3}\right)$, seis diretores de curso $\left(\mathrm{DC}^{4}\right)$, três diretores das Unidades Orgânicas $\left(\mathrm{DUO}^{5}\right)$, dois técnicos de Ação Social $\left(\operatorname{TAS}^{6}\right)$ e uma bibliotecária $\left(\mathrm{TB}^{7}\right)$.

\section{Análise e discussão dos resultados}

\section{Presença e inclusão dos estudantes com NEE}

A presença de ENEE na Universidade do Algarve não é uma novidade para os entrevistados (P1, P6, DC1, DC4, DC5, DUO2, DUO3, TAS1, TAS2, TB), mas estes reconhecem que a sua presença ainda tem pouca expressão e é "uma minoria" (P2, DC1, DC4, DC5, DUO3, TAS2, TB). Assinale-se que, segundo os entrevistados, esta minoria tem vindo a aumentar (P1, P2, P9), o que pensam estar relacionado com a possibilidade que tem vindo a ser dada a estes estudantes de terem apoio e respostas diferenciadas. Dos diretores das Unidades Orgânicas entrevistados, dois consideram que, de forma geral, ao longo dos últimos anos tem havido uma perda de estudantes que entram no ES, sendo que este facto acaba também por afetar o número de ENEE que se candidata (DUO1, DUO3). Este cenário é semelhante ao referido por Curado e Oliveira (2010), que também constatam um decréscimo do número de ENEE inscritos no $1 .^{\circ}$ ano, dos cursos de $1 .^{\circ}$ ciclo, entre 2003 e 2009, na Universidade de Lisboa. Dados da Direção-Geral do Ensino Superior (2012) revelam um aumento de candidaturas de estudantes ao ES pelo Contingente Especial para Candidatos com Deficiência Física ou Sensorial em 2009, seguindo-se, até 2011, um progressivo decréscimo. Assinale-se que estudos recentes referem que, em muitos países, embora de forma lenta, o número dos ENEE no ES tem vido a aumentar (Abu-Hamour, 2013; Berggren et al., 
2016; Cabral et al., 2015; Couzens et al., 2015; European Commission, 2012; Fossey et al., 2017; Gumbi et al., 2015).

Encontrámos nas palavras de alguns entrevistados a menção da ausência de uma política definida especificamente para promover o acesso e a equidade no ES a estes cidadãos: "só quando aparece um aluno" (P3) é que se pensa em educação inclusiva. Esta perspetiva é também partilhada por Castanheira (2013) e Fernandes e Almeida (2007). Neste sentido, alguns participantes (P1, DC2, DUO1, TAS2) referem a necessidade de se adotar uma política e estratégias de atuação específicas para a promoção do acesso e equidade no ES, assim como a criação de mecanismos que garantam as condições favoráveis à permanência destes estudantes nas universidades. Estas são condições de sucesso corroboradas na literatura (Fernandes \& Almeida, 2007; FOTIM, 2011; Kimball, Wells, Ostiguy, Manly, \& Lauterbach 2016; Porfírio et al., 2016; Venville et al., 2014; Wessel, Jones, Markle, \& Westfall, 2009).

A presença dos ENEE no ES é encarada como um "direito, como o de qualquer cidadão" (P1,P5, P6, DC1, DC4, DC5, DUO1, TAS2). Este direito é entendido pela maioria dos docentes como uma "mais-valia" para as instituições de Ensino Superior, por estes estudantes "serem uma lição de vida, por enriquecerem a instituição pela diversidade e por serem uma motivação para os outros estudantes" (P6). Neste reconhecimento, a maioria dos entrevistados afirma estar a trabalhar para garantir o sucesso académico e a inclusão dos ENEE (P4, P5, P3, P6, DC1, DC2, DC3, DC4, DC5, DC6, DUO1, TAS1): "no geral queremos é ajudar" (DC1); "dar resposta positiva a todos os casos que têm aparecido" (DC4); "estamos a fazer com que o aluno não seja esquecido" (P6).

Existe na UAlg uma preocupação destes atores sociais em garantir as condições necessárias para incluir os ENEE (P3, P4, P5, P6, DC3, DC4, TB, TAS2), reconhecendo que "existe o espírito [de inclusão] nesta universidade" (DC4) e que, na sua essência, a afirmação "na minha universidade vive-se a inclusão" é uma realidade, "se bem que nem tudo esteja a funcionar a 100\%" (DC3). Os entrevistados referem, contudo, que a inclusão continua dependente, fundamentalmente, da boa vontade dos docentes, e não de uma política concreta (P1, P2, P3), nem sempre existindo os meios e condições adequados para responder às necessidades dos alunos. É neste sentido que 
o DC3 refere a carência de "recursos físicos, pedagógicos", e ainda "turmas grandes, pouco tempo para trabalhar com estes estudantes, dificuldades de interação com a turma e docentes". Estes resultados são concordantes com muitos estudos que referem não ser suficiente a boa vontade para que a inclusão dos ENEE seja uma realidade e que é necessário ultrapassar barreiras no que se refere às acessibilidades, mas também as barreiras atitudinais e que têm impacto na inclusão destes estudantes (Abu-Hamour, 2013; Berggren et al., 2016; Cabral et al., 2015; Fossey et al., 2017; Gumbi et al., 2015; Venville et al., 2014). Não obstante o esforço em prol da inclusão, reconhece-se que "para a instituição e às vezes para o próprio aluno acaba por ser muito difícil e é frustrante ... quando o aluno quer fazer uma coisa, mas depois não há como" (DC1), pelo que "há ainda um caminho que temos de percorrer" (DC5).

Um dos diretores de curso (DC2) levanta ainda a questão da "adequação do curso ao perfil do estudante com NEE" pelas implicações que tem no seu sucesso, quer académico quer profissional. O problema coloca-se quando a escolha do curso se revela desadequada pelas características e limitações advindas da problemática apresentada pelo estudante, porque por vezes estas revelam-se incompatíveis com o futuro exercício profissional. É o caso da perturbação do espectro do autismo, em particular da síndrome de Asperger, com implicações ao nível da interação social e com uma previsível incompatibilidade no exercício de profissões que obriguem a uma forte interação social, como o ensino e as profissões na área da saúde:

um individuo que deliberadamente assume que não se relaciona com ninguém, é ele que nos diz isto, tem esta consciência... Dizer querer ser radiologista ou psicólogo não me parece acertado! Se calhar... estaria muito melhor noutra área em que não precisasse ter uma relação, se calhar. (DC2)

Importa ainda assinalar que esta preocupação não parece decorrer de uma postura excludente, mas inclusiva, pois como refere ainda o diretor de curso: "não é que eu me queira ver livre do E21, de forma nenhuma, de forma nenhuma ... mas ele vai ter problemas mais à frente" (DC2).

$\mathrm{Na}$ UAlg, a história recente no apoio institucional formal pode explicar por que, apesar do número crescente de ENEE, os entrevistados apresentam a perceção de que a universidade ainda não está totalmente preparada para acolher estes estudantes (P1, P2, P3, DC1, DC3, DC6, DUO1, TAS1, TAS2). 
Em particular, assinala-se algum desconhecimento dos docentes (P4, P5, P3,P2, P1) e diretores de curso (DC1) sobre o serviço de apoio aos estudantes com NEE, sendo apontada por estes a "falta de serviços de apoio", o que, segundo alguns entrevistados, "implica a necessidade de um apoio mais atento por parte das famílias" (P1, P3 DUO1, DUO3, TAS1).

\section{Serviços e recursos institucionais}

À data da aplicação das entrevistas, o recém-criado Gabinete de Apoio ao Estudante com NEE da Universidade do Algarve (GAENEE) iniciava as suas funções, pelo que parece justificar-se que alguns dos entrevistados refiram não conhecer ainda o serviço (P4, P5, P3,P2, P1, DC1) e o Estatuto criado para formalizar os apoios que podem ser disponibilizados aos estudantes (DC1, DC2, DC5), sugerindo os entrevistados uma maior divulgação (P2, DC1, DC3, DC4) para uma maior "visibilidade" (P4, TB). Os técnicos de Ação Social destacam a importância do GAENEE, referindo que "o gabinete está em todo o lado, encontrando-se organizado de uma forma simples e eficaz, acessível a partir de qualquer ponto do campus universitário" (TAS1, TAS2). Para os que referem conhecer o trabalho realizado pelo GAENEE, este é apresentado como "uma mais-valia" (DC1, DC2, DC3, DC4, DC5, P4, P1, P6), tal como referido em outros estudos que assinalam que a existência dos serviços de apoio a estes estudantes é crucial (Couzens et al., 2015; Fossey et al., 2017; FOTIM, 2011; Harrison, Hemingway, Sheldon, Pawson, \& Barnes, 2009).

Com a criação do Gabinete de Apoio, "o diretor de curso passou a ter um papel de ligação" (DC4, DC5) com o ENEE e os docentes, veiculando a informação do Gabinete, nomeadamente informando quais os estudantes com Estatuto e quais as medidas que Ihes são aplicadas, mas também procurando junto do GAENEE respostas às dúvidas e dificuldades sentidas pelos docentes.

A tomada de conhecimento da presença de ENEE fazia-se, segundo alguns entrevistados, à data da investigação, de diversas formas, como "no decorrer das aulas" (P4, P6, DC6, DC1), através de "colegas" (DC1, DC3, DC5), pelo "diretor do curso" (P2, P3, P6, DC1, DC3, DC5), nas "secretarias" (DUO1), no "Conselho Pedagógico" (DUO3), na "Escola/Faculdade/ Departamento" (DUO1), através do "contacto direto" (TAS1, TAS2, TB), por 
"informação do ensino secundário" (P2, P1) e pela "observação de alguns comportamentos desadequados" (DC1).

É de salientar que muitos dos entrevistados (P3, P4, P6, DC1, DC3, DC5, DUO1, DUO3, TAS1, TAS2) assinalam que nem todos os ENEE estão predispostos a partilhar a sua problemática à chegada ao ES. Estes resultados acompanham o que é expresso na literatura da área, em que se refere que, com receio do estigma e da discriminação, muitos são os estudantes que optam por não pedir apoio e permanecer no anonimato (Berggren et al., 2016; Couzens et al., 2015; Fernandes \& Almeida, 2007; Fossey et al., 2017; FOTIM, 2011; French et al., 2014; Gumbi et al., 2015; Nolan et al., 2015), equivalendo a que, nestes casos, não se consiga evitar a ocorrência de situações de tomada de conhecimento na sala de aula.

Uma necessidade ainda identificada é a articulação entre os ensinos secundário e superior, sugerindo um maior acompanhamento no processo de transição entre estes níveis de ensino. O contributo desta articulação para o sucesso académico do ENEE no ES é confirmado por diversos estudos, que chamam atenção para a importância de existir um plano de transição estruturado que evite uma rutura no itinerário do aluno (Castanheira, 2013; Fernandes \& Almeida, 2007; FOTIM, 2011).

Os entrevistados reconhecem também "não ter formação" (P4, P5, P3, P2, P1, DC1, DC2, DC3, DC4, DC6) que os habilite a trabalhar/interagir de forma adequada com este público. A necessidade de formação, sentida como "necessária e importante" (P1, P3, DC1, DC2, DC3, DC4, DC5, DUO1, DUO2, DUO3, TAS1, TAS2, TB), é considerada como uma das condições para o sucesso académico dos ENEE em diversos estudos (Abu-Hamour, 2013; Couzens et al., 2015; Fernandes \& Almeida, 2007; Fossey et al., 2017; FOTIM, 2011; French et al., 2014; Gonçalves \& Cardoso, 2010; Gumbi et al., 2015; Harrison et al., 2009; Johnson, Becker, Estrada, \& Freeman, 2015; Mamah et al., 2011; Meira \& Pereira, 2017; Morgado et al., 2016). É também sugerida pelos diretores de curso a necessidade de "dar formação aos colegas" dos ENEE para que estes saibam lidar com estas situações, ou "campanhas de sensibilização" junto da comunidade académica para que "haja mais tolerância no ES" (DC5). De acordo com este diretor de curso, "na universidade o ambiente demasiado competitivo faz as pessoas não se preocuparem com os outros" (DC5), sublinhando, contudo, que este é um 
aspeto que "faz parte da formação . . . da educação das pessoas" (DC5). Outro diretor de curso argumenta que

a formação seria uma forma de contrariar a imagem prevalecente da pessoa com deficiência como alguém incapaz, contrapondo uma outra que mostre que estas pessoas têm capacidades para ter uma vida normal, para terem uma profissão, para trabalhar e para serem independentes, autónomas . . . se tiverem acesso à educação e à formação. (DC4)

\section{Infraestruturas e acessibilidades}

São igualmente apontadas algumas falhas e problemas nas infraestruturas e acessibilidades por todos os intervenientes neste cenário educativo, resultados corroborados em diversos estudos nacionais e internacionais (Berggren et al., 2016; Couzens et al., 2015; Fossey et al., 2017; French et al., 2014; Gonçalves \& Cardoso, 2010; Gumbi et al., 2015; Harrison et al., 2009; Ndlovu \&Walton, 2016; Santos et al., 2015; Venville et al., 2014). Por exemplo, "no acesso às salas de aula" (P2, P4, P6, DC4), em particular "a anfiteatros, auditórios e bibliotecas" (P6, TB), a "inexistência/desadequação de elevadores ou em situação prolongada de avaria" (P2, DC1, TAS2), a "falta de casas de banho adaptadas" (P6, TAS2) e "salas de aula desadequadas" (P6).

Os entrevistados assinalam ainda a "ausência ou poucos locais de estacionamento para alunos com deficiência" (DUO3) e "obstáculos na acessibilidade no campus" (DUO1, DUO2). Estes problemas são identificados não apenas nos edifícios mais antigos, mas igualmente nas estruturas construídas mais recentemente, em "desrespeito pela legislação existente sobre esta matéria" (DC1, DC5).

Para obviar estas situações, a Universidade do Algarve tem vindo a realizar diversas obras e adaptações para criar melhores condições de acessibilidade nos espaços dos campi, referindo-se, contudo, que é "à medida que vão aparecendo alunos é que se vão fazendo remendos" (P2, DC1, DC5). Dois dos diretores de curso acrescentam que "quando não é possível fazer obras ou introduzir equipamentos para melhorar a acessibilidade às aulas, resolve-se a situação mediante uma gestão dos espaços e dos horários" (DC3, DC4). 


\section{Dimensão pedagógica}

Apesar da falta de formação, os testemunhos de docentes e diretores de curso revelam que, quando lidam com os alunos com NEE, procuram dar resposta às suas necessidades procedendo a adequações pedagógicas diversas, nomeadamente a "adequação do processo de avaliação" (P3, P5, P6, DC4, DC6), a "adequação dos instrumentos de avaliação" (P2, P3, DC1, DC4), a "adequação na lecionação das aulas" (P1, P2, P3, P5, P6, DC1, DC2, DC4), "dando apoio fora da sala" (P2, P3, P4, P5, DC4), a "utilização de material específico" (P1, P2, P3, P6, DC1) e a "construção de materiais adequados" (P2, P3, P5). De acordo com os entrevistados, este trabalho visa "colmatar as dificuldades para eles poderem lá chegar" (P3), "no reconhecimento de que eles não estão a ser beneficiados ou facilitados, mas simplesmente estamos a ajustar a realidade... Portanto, estamos apenas a dar-Ihes as mesmas oportunidades" (DC4).

Não obstante, também são relatadas atitudes menos tolerantes, como por exemplo a de um dos diretores de curso, que afirma:

pois, mas não é fácil... Tenho uma sala cheia... assim não dá para adotar uma estratégia para um aluno!. . . . A gente faz o melhor que pode . . . mas esses alunos, inseridos em turmas de 30 ou mais, têm direito ao mesmo tempo que têm os outros. (DC6)

O impacto das adequações pedagógicas no sucesso académico é "considerado positivo" (P1, P2, P3, P4, P5, P6, DC1), contrariando o insucesso e posterior abandono do curso, o que vai ao encontro de alguns estudos neste domínio (Moriña, Cortés, \& Melero, 2013; Ndlovu \& Walton, 2016; Nolan et al., 2015; Sachs \& Schreuer, 2011; Venville et al., 2014). De facto, quando existe adequação pedagógica estes alunos podem apresentar resultados académicos tão bons quanto os dos colegas (P3, P4, DC1, DC4, DC6, DUO1). É referido por um dos entrevistados o exemplo de um estudante com paralisia cerebral que "fez o curso lindamente e depois acabou por fazer o Doutoramento cá também... Foi um Doutoramento em Engenharia Informática e ficou como investigador, num grupo de trabalho" (DC1).

Todavia, reconhece-se que estes alunos precisam de ser mais apoiados do que os restantes (P1, P4, P5, DC6, TAS2) e que podem reprovar ou desistir mais facilmente, sobretudo "quando não existe" apoio (DC1, DC4, DC5, TAS1, TAS2). Outro fator importante para o sucesso ou insucesso 
académico é "a atitude do próprio estudante com NEE" (DC3, P2, P3, DUO1), fator também identificado na literatura (Gonçalves \& Cardoso, 2010; Sachs \& Schreuer, 2011; Wessel et al., 2009).

Assinale-se, contudo, ainda alguns testemunhos reveladores das dificuldades em aceitar os ENEE no ES:

se, por exemplo, o aluno fosse invisual, tu não lhe podias dar um texto para ele ler, porque ele não ia ler . . . tem um estatuto que lhe permite não ler o texto naquele formato. E o outro ficou muito espantado: 'Então mas eu agora tenho que adaptar o método consoante....?'. 'Claro que tens que adaptar o método . . . se o aluno fosse cego, que não conseguisse ler, tinhas que... pôr em Braille ou contar com um método diferente, não é?'. . . . E o meu colega ficou calado...Viu que eu tinha razão... não é?. . . Portanto, só para dizer que ainda há muita gente que não está preparada para lidar com estas situações, não é flexível. (P4)

A análise a este relato permite entender a perceção de que o ENEE possa ser incapaz para seguir o ES, testemunho também referido por Gonçalves e Cardoso (2010) e que expressa a falta de formação de alguns docentes. Todavia, e apesar da presença de discursos que se podem considerar ainda discriminatórios e negativos quanto à capacidade dos ENEE e ao direito que estes têm de frequentar o ES, os testemunhos dos docentes da UAlg realçam uma progressiva sensibilização, de que é exemplo o referido por um dos docentes: "porque os professores universitários hoje em dia já são pessoas mais novas... já não é aquele clássico universitário" (P1).

Do estudo efetuado, constata-se que as principais complexidades sentidas com os ENEE são a "dificuldade em lidar com comportamentos desadequados na sala de aula" (P2, P3, P4, DC6, TAS2), a "dificuldade em obter recursos adequados da universidade"'" (P1, P3, DC1, TAS1), a "perda de apoios quando transitam para o ES" (P1, P2), o "não conseguir/saber responder às necessidades do aluno" (P3, DC1, DC4). São ainda referidas as "dificuldades associadas à problemática" (P2, P3, P4, DC1, DC2, DC3, DC6, TAS1, TAS2), a "dificuldade ou impossibilidade de fazer a unidade curricular devido à problemática do estudante", e que está diretamente relacionada com a "questão da adequação do curso ao perfil do estudante" (DC1, DC2), a "recusa do aluno em colaborar" (P2, P3, P5, DC5), o "desejo de anonimato por parte do estudante, que não requer o Estatuto" (P2, P3, P5, P6, DC1, DC3, DC4, DC5, TAS2) como forma de evitar a estigmatização e a discriminação. Assinale-se que o desejo de anonimato é identificado em diversos estudos 
(Berggren et al., 2016; Couzens et al., 2015; Fernandes \& Almeida, 2007; Fossey et al., 2017; FOTIM, 2011; French et al., 2014; Gumbi et al., 2015; Madriaga \& Goodley, 2010; Nolan et al., 2015).

\section{Socialização, inclusão académica e social}

No que se refere às relações estabelecidas, os entrevistados, na sua maioria, expressam a "existência de uma boa interação com estes estudantes" (P1, P2, P3, P4, P5, P6, DC1, DC3, DC4, DC5, DC6, DUO2, TAS2), que não se limita à sala de aula, indiciando a existência de uma inclusão positiva. No entanto, encontram-se também testemunhos de problemas de comunicação que levaram à "exclusão do aluno/a" por parte dos colegas ou, em alguns casos, à "autoexclusão" (P2, P3, P4, TAS2), ou de "uma fraca ou ausência de interação" (DC2), que, por vezes, se atribuiu ao isolamento do próprio ENEE (DC1).

De assinalar que, no presente estudo, não só não foram relatadas ocorrências que explicitassem situações de discriminação dos estudantes com NEE, como foi referido o papel decisivo desempenhado pelos colegas de turma no processo de inclusão: "eu acho que os colegas tiveram um papel muito, muito importante durante todo o desenrolar do percurso dele aqui na instituição" (DUO3). Este papel constitui uma importante base de apoio e suporte emocional, quer em contexto de sala de aula (ex.: trabalhos de grupo), quer no acesso a outros espaços do ambiente académico (ex.: Serviços Académicos, cantinas, bares). No mesmo sentido se encontram os resultados apresentados por Wessel e colaboradores (2009) e Hughes (2015), que concluíram que a participação dos ENEE em atividades e grupos de apoio nos campi aumenta o sentido de pertença e de identificação com a comunidade académica, eleva a autoestima, afeta positivamente o sucesso académico e previne a reprovação e o abandono.

\section{Conclusões}

Os resultados encontrados na presente investigação permitem constatar que as experiências dos estudantes com NEE em termos de acesso e condições globais para permanecer no ES são ainda marcadas por diversas dificuldades e constrangimentos. 
Os testemunhos dos docentes e pessoal não-docente entrevistados revelam que continuam a existir inúmeras barreiras físicas e arquitetónicas que condicionam a mobilidade e o acesso, não só nos campi, mas também aos anfiteatros e salas de aula. Igualmente referenciados são a falta de equipamentos, de recursos educativos, de formação e de um maior apoio pedagógico aos docentes.

Também as respostas institucionais e os serviços são referidos como apresentando falhas, nomeadamente ao nível da articulação entre o Ensino Secundário e o Ensino Superior e de acompanhamento no processo de transição entre os dois níveis de ensino. Apesar de se procurar responder às necessidades e exigências destes estudantes, muito do que se faz resulta da "boa vontade", salientando-se a inexistência de uma política institucional direcionada para a inclusão no Ensino Superior. A assunção desta política permitiria um trabalho mais direcionado e concertado, nomeadamente no combate às barreiras atitudinais, que continuam a ser um dos principais obstáculos para a inclusão. Os testemunhos dos entrevistados e a pesquisa já realizada neste âmbito apontam que as iniciativas não podem, nem devem, depender exclusivamente das iniciativas e boa vontade de cada instituição de ES e que deve ser desenvolvida uma legislação nacional que dê resposta às necessidades destes estudantes. A legislação deve contemplar a adequação dos serviços e recursos institucionais, as infraestruturas e acessibilidades, as questões relacionadas com a dimensão pedagógica e a socialização e a inclusão académica e social dos ENEE, que cada vez estão mais presentes no ES.

Face a estas constatações, é importante, como principal desafio, que, à luz do paradigma do Desenho Universal, as instituições de ensino integrem uma política que incorpore os conceitos e princípios do Desenho Universal para a Aprendizagem, para que possam responder eficazmente às necessidades de todos os estudantes, e especialmente dos estudantes com necessidades educativas especiais (Domings, Crevecoeur, \& Ralabate, 2014; Hitchcock, Meyer, Rose, \& Jackson, 2002; Katz, 2014).

No sentido de guiar a mudança institucional no ES, apresentam-se algumas recomendações, que devem assentar em três vertentes principais:

1) Políticas educativas: sensibilização, informação e formação dirigidas a toda a comunidade académica (professores, pessoal 
não-docente e estudantes), desmistificando as problemáticas e as atitudes discriminatórias e fornecendo indicações para um atendimento e apoio eficazes aos ENEE;

2) Serviços e recursos institucionais: os edifícios, instalações e espaços das universidades, incluindo os espaços virtuais, os serviços, procedimentos e informações, devem ser acessíveis, garantindo que nenhum membro da comunidade universitária encontre barreiras e/ou constrangimentos à sua participação na vida universitária. Deve-se ainda proporcionar o acesso a tecnologias de apoio necessárias à inclusão que possibilitem um melhor desempenho e autonomia de todos os estudantes.

3) Acesso à informação e ao apoio do estudante com NEE: o acesso à comunicação e informação é um direito que deve ser garantido pelas instituições de Ensino Superior a todos os estudantes. Após a formação universitária, é ainda essencial que a universidade estabeleça contactos e parcerias com os serviços de orientação laboral, entidades públicas e privadas, para impulsionar políticas de inclusão laboral.

Como limitações da presente investigação, importa começar por referir que a inclusão encerra muitas e diversas complexidades, atendendo aos diversos fatores implicados e aos diversos atores em cena. A abordagem por que optámos no presente artigo, nomeadamente as entrevistas aos docentes e ao pessoal não-docente, representa apenas uma das dimensões desta realidade, pelo que esta constitui a principal limitação do estudo apresentado, uma vez que não dá voz a outros intervenientes, particularmente aos estudantes com NEE e aos seus colegas. O facto de os dados obtidos serem apenas referentes a uma universidade poderá constituir também uma limitação, pelo que se torna urgente a realização de mais estudos de forma a podermos ter uma visão mais abrangente da inclusão no Ensino Superior, das dificuldades que ainda permanecem e do que deve ser feito para se caminhar para uma Universidade para Todos.

Espera-se, no entanto, que os resultados e as reflexões da presente investigação possam contribuir para que a comunidade académica e os gestores políticos se consciencializem de que, se muito já foi feito, muito ainda permanece por fazer para que as universidades assegurem o acesso, a 
participação e o sucesso de todos os alunos, combatendo-se deste modo qualquer forma de exclusão e caminhando-se para uma sociedade inclusiva que a todos respeite e valorize e que ofereça equidade de oportunidades para que cada pessoa possa exercer a sua autonomia e autodeterminação.

\section{Notas}

1 Este artigo é financiado por Fundos Nacionais através da FCT - Fundação para a Ciência e a Tecnologia, no âmbito do projeto "Estudantes não-tradicionais no Ensino Superior: Investigar para guiar a mudança institucional" (IVC-PEC/4886/2012).

2 No ano letivo em que foi realizado o estudo, os dados reportam-se a 16 estudantes, incluindo Deficiência Auditiva (1); Perturbação Emocional (1); Dislexia (1); Doença Neurológica (1); Deficiência Motora (9); Perturbação do Espectro do Autismo (3).

3 Os docentes são codificados como P1, P2, P3, P4, P5 e P6.

4 Os diretores de curso são codificados como DC1, DC2, DC3, DC4, DC5 e DC6.

5 Os diretores das Unidades Orgânicas são codificados como DUO1, DUO2, DUO3.

6 Os técnicos de Ação Social são codificados como TAS1 e TAS2.

7 A bibliotecária é codificada como TB.

\section{Referências}

Abreu, S. M. (2011). Alunos com necessidades educativas especiais: Estudo exploratório sobre a inclusão no Ensino Superior (Dissertação de Mestrado não publicada). Universidade da Madeira, Funchal. Disponível em: http://hdl.handle.net/10400.13/330.

Abu-Hamour, B. (2013). Faculty attitudes toward students with disabilities in a public university in Jordan. International Education Studies, 6(12), 74-81. doi:10.5539/ies.v6n12p74.

Antunes, A. P., \& Faria, C.P. (2013). A universidade e a pessoa com necessidades especiais: Estudo qualitativo sobre perceções de mudança social, institucional e pessoal. Indagatio Didactica, 5(2), 474-488. http://revistas.ua.pt/index.php/ ID/article/view/2467/2384.

Antunes, A. P., Faria, C. P., Rodrigues, S. E., \& Almeida, L. S. (2013). Inclusão no Ensino Superior: Percepções de professores em uma universidade portuguesa. Psicologia em Pesquisa, 7(2), 140-150.

Bardin, L. (2009). Análise de conteúdo. Lisboa: Edições 70.

Berggren, U. J., Rowan, D., Bergbäck, E., \& Blomberg, B. (2016). Disabled students' experiences of higher education in Sweden, the Czech Republic, and the United States - A comparative institutional analysis. Disability \& Society, 31(3), 339356. doi: 10.1080/09687599.2016.1174103. 
Bisol, C. A., \& Valentin, C. B. (2012). Desafios da inclusão: Uma proposta para a qualificação de docentes no Ensino Superior via tecnologias digitais. Revista Portuguesa de Educação, 25(2), 263-280. http://www.scielo.mec.pt/pdf/rpe/ v25n2/v25n2a12.pdf

Cabral, L. S. A., Mendes, E. G., de Anna, L., \& Ebersold, S. (2015). Academic and professional guidance for tertiary students with disabilities: Gathering best practices throughout European universities. Open Journal of Social Sciences, 3(9), 48-59. doi: 10.4236/jss.2015.39008

Campbell, T., Fontes, E., Hemingway, L., Soorenian, A., \& Till, C. (2008). Disability studies: Emerging insights and perspectives. Leeds: The Disability Press.

Capucha, L. (2010). Inovação e justiça social. Políticas activas para a inclusão educativa. Sociologia, Problemas e Práticas, 63, 25-50. http://sociologiapp. iscte-iul.pt/pdfs/10163/10181.pdf

Castanheira, L. (2013). Integração de alunos com necessidades educativas especiais no Ensino Superior: A evidência de um percurso. In B. D. Silva, L. S. Almeida, A. Barca, M. Peralbo, A. Franco, \& R. Monginho (Orgs.), Atas do XII Congresso Internacional Galego-Português de Psicopedagogia (pp. 5588-5595). Braga: Universidade do Minho. http://webs.ie.uminho.pt/xiigp/at11.pdf

Couzens, D., Poed, S., Kataoka, M., Brandon, A., Hartley, J., \& Deb, K. (2015). Support for students with hidden disabilities in universities: A case study. International Journal of Disability, Development and Education, 62(1), 24-41. doi: 10.1080/1034912X.2014.984592

Curado, A. P., \& Oliveira, V. (2010). Estudantes com necessidades educativas especiais na Universidade de Lisboa. Gabinete de Garantia da Qualidade. Lisboa: Universidade de Lisboa. www.opest.ul.pt/pdf/ENEE_UL.pdf

Direção-Geral do Ensino Superior (2012). Concurso nacional de acesso e ingresso no Ensino Superior público: Contingente especial para portadores de deficiência física ou sensorial: 2007-2011. Lisboa: Direção Geral do Ensino Superior. Disponível em: http://www.dges.mctes.pt/DGES/pt/Estudantes/Acesso/ Estatisticas/EstudosEstatisticas/

Domings, Y., Crevecoeur, Y. C., \& Ralabate, P. K. (2014). Universal design for learning. Meeting the needs of learners with autism spectrum disorders. In K. I. Boser, M. S. Goodwin, \& S. C. Wayland (Eds.), Technology tools for students with autism. Innovations that enhance independence and learning (pp. 21-41). Baltimore: Paul Brookes Publishing.

European Commission (2012). Education and disability/special needs - Policies and practices in education, training and employment for students with disabilities and special educational needs in the EU. Disponivel em: http://www.nesse.fr/ nesse/activities/reports

Faria, C. P. (2012). Inclusão de alunos com necessidades educativas especiais no Ensino Superior: Estudo exploratório sobre as perceções dos docentes (Dissertação de Mestrado) Universidade da Madeira, Funchal. Disponível em: http://repositorio.uma.pt/handle/10400.13/509

Fernandes, E., \& Almeida, L. (2007). Estudantes com deficiência na universidade: Questões em torno da sua adaptação e sucesso académico. Revista de Educação Especial e Reabilitação, 14(7), 12-24. 
Fossey, E., Chaffey, L., Venville, A., Ennals, P., Douglas, J., \& Bigby, C. (2017). Navigating the complexity of disability support in tertiary education: Perspectives of students and disability service staff. International Journal of Inclusive Education, 21(8), 822-832. doi: 10.1080/13603116.2017.1278798

Foundation of Tertiary Institutions of the Northern Metropolis (2011). Disability in higher education. Project report. Johannesburg: Disability Management Services. Disponível em: http://www.students.uct.ac.za/usr/disability/reports/annual_ report_10_11.pdf

Fragoso, A., Ribeiro, C. M., Quintas, M. H., Fonseca, H. Marques, J. F., Santos, L., ...Gonçalves, R. (2015). Estudantes não-tradicionais no Ensino Superior: Investigar para guiar a mudança institucional - Relatório final do projeto (Ref.: PTDC/IVC-PEC/4886/2012). Universidade do Algarve \& Universidade de Aveiro [Documento policopiado].

França, S. D. (2014). Inclusão de alunos com NEE no Ensino Superior: Um estudo de caso na Universidade Estadual de Montes Claros (Unimontes) (Tese de Doutoramento). Universidade de Trás-os-Montes e Alto Douro, Vila Real.

French, E., Summers, J., Kinash, S., Lawson, R., Taylor, T., Herbert, J., ...Hall, C. (2014). The practice of quality in assuring learning in higher education. Quality in Higher Education, 20(1), 24-43. doi: org/10.1080/13538322.2014.889432

Gonçalves, I., \& Cardoso, P. M. S. (2010). Percepção de barreiras da carreira: Estudo com estudantes universitários com incapacidade. International Journal of Developmental and Educational Psychology, XXI, 495-503. http://hdl.handle.net $/ 10174 / 2515$

Gonçalves, T., Borges, M. L., \& Martins, M. H. (2014). Students with special needs education: Reflections around inclusion. In S. O'Rouke, A. P. L. Martins, T. P. Gumpel, A. Cruz-Santos, A. P. S. Pereira, A. S. Serrano, \& H. J. RodriguezHernandez (Orgs.), Proceedings of Braga 2014 Embracing Inclusive Approaches for Children and Youth with Special Education Needs Conference (pp. 156-160). Braga: CIED - Centro de Investigação em Educação, Instituto de Educação, Universidade do Minho.

Grupo de Trabalho para o Apoio a Estudantes com Deficiência no Ensino Superior. (2014). Inquérito nacional sobre os apoios concedidos aos estudantes com necessidades educativas especiais no Ensino Superior. Disponível em: http://gtaedes.ul.pt/gtaedes/inq_superior

Gumbi, D., Cekiso, M., Gqweta, Z., Makiwane, B., Majeke, L., Bojanyana, N. ...Ludabi, W. (2015). An exploration of challenges related to inclusion of students with disabilities at a university of technology in South Africa. International Journal of Educational Sciences, 8(2), 261-266.

Harrison, M., Hemingway, L., Sheldon, A., Pawson, R., \& Barnes, C. (2009). Evaluation of provision and support for disabled students in higher education. Bristol: The Higher Education Funding Council for England. Disponível em: http://www.hefce.ac.uk/pubs/rdreports/2009/rd24_09/

Hitchcock, C., Meyer, A., Rose, D., \& Jackson, R. (2002). Providing new access to the general curriculum: Universal design for learning. Teaching Exceptional Children, 35(2), 8-17. Disponível em: https://www2.bc.edu/alecpeck/Jackson\%20UDL.pdf 
Hughes, K. (2015). The social inclusion meme in higher education: Are universities doing enough? International Journal of Inclusive Education, 19(3), 303-313. doi.org/10.1080/13603116.2014.930518

Johnson, L., Becker, S. A., Estrada, V., \& Freeman, A. (2015). NMC Horizon Report: 2015, Higher Education Edition. Austin, Texas: The New Media Consortium. Disponível em: https://www.nmc.org/publication/ nmc-horizon-report-2015higher-education-edition/

Katz, J. (2014). Implementing the three block model of universal design for learning: Effects on teacher's self-efficacy, stress, and job satisfaction in inclusive classroom K-12. International Journal of Inclusive Education, 19(1), 1-20. doi: 10.1080/13603116.2014.881569

Kimball, E., Wells, R., Ostiguy, N., Manly, C., \& Lauterbach, A. (2016). Students with disabilities in higher education: $\mathrm{A}$ review of the literature and an agenda for future research. In M. Paulsen (Ed.), Higher education: Handbook of theory and research (pp. 91-156). Dordrecht: Springer International Publishing Switzerland. doi: 10.1007/978-3-319-26829-3_3

Madriaga, M., \& Goodley, D. (2010). Moving beyond the minimum: Socially just pedagogies and Asperger's syndrome in UK higher education. International Journal of Inclusive Education, 14(2), 115-131. doi: 10.1080/1360311 0802504168

Mamah, V., Deku, P., Darling, S., \& Avoke, S. (2011). University teachers' perception of inclusion of visually impaired in Ghanaian Universities. International Journal of Special Education, 26(1), 70-79. http://files.eric.ed.gov/fulltext/EJ921189.pdf

Martins, M. H., Borges, M. L. Gonçalves, T., \& Fonseca, H. M. A. C. (2014). Estudantes com mobilidade reduzida no Ensino Superior: Testemunhos na primeira pessoa! In M. J. Carvalho, A. Loureiro, \& C. A. Ferreira (Orgs.), Atas do XII Congresso da Sociedade Portuguesa de Ciências da Educação. Ciências da Educação: Espaços de investigação, reflexão e ação interdisciplinar (pp. 1804-1814). Vila Real: Universidade de Trás-os-Montes e Alto Douro.

Meira, C. E. B., \& Pereira, A. (2017). Direitos das pessoas com deficiência: Uma análise sobre acessibilidade no Ensino Superior. In M. L. Borges, C. Luísa, \& H. Martins (Orgs.), Atas do II Congresso Internacional Direitos Humanos e Escola Inclusiva: Múltiplos olhares (pp. 126-134). Algarve: Universidade do Algarve. Disponível em: http://sapientia.ualg.pt/handle/10400.1/8999

Melo, L. V., \& Martins, M. H. (2016). Legislação para estudantes com deficiência no Ensino Superior no Brasil e em Portugal: Algumas reflexões. Acta Scientiarum - Education, 38(3), 259-269. doi: 10.4025/actascieduc.v38i3.30491

Moreira, L. C., Bolsanello, M. A., \& Seger, R. G. (2011). Ingresso e permanência na universidade: Alunos com deficiências em foco. Educar em Revista, 41, 125143. http://revistas.ufpr.br/educar/article/view/25006/16754

Morgado, B., Melero, N., Molina, V., \& Cortés-Vega, M. D. (2016). Inclusive university classrooms: The importance of faculty training. In J. Domenech, M. C. VincentVela, R. Peña-Ortiz, E. de la Poza, \& D. Blazquez (Eds.), 2nd International Conference on Higher Education Advances, HEAd'16 (pp. 182-189). València: Universitat Politècnica de València. http://www.headconf.org/wp-content/ uploads/pdfs/2630.pdf 
Moriña, A., Cortés, M. D., \& Melero, N. (2013). Inclusive curricula in Spanish higher education? Students with disabilities speak out. Disability \& Society, 29, 44-57. doi: 10.1080/09687599.2013.769862

Ndlovu, S., \& Walton, E. (2016). Preparation of students with disabilities to graduate into professions in the South African context of higher learning: Obstacles and opportunities. African Journal of Disability, 5(1), 8 pages. http://www.ajod.org/ index.php/ajod/article/view/150/408

Nolan, C., Gleeson, C., Treanor, D., \& Madigan, S. (2015). Higher education students registered with disability services and practice educators: Issues and concerns for professional placements. International Journal of Inclusive Education, 19(5), 487-502. doi: 10.1080/13603116.2014.943306

Patton, M. Q. (1990). Qualitative evaluation and research methods. London: Sage.

Porfírio, J. A., Gronita, J., Carrilho, T., \& Silva, H. V. (Coords.). (2016). Ensino Superior para pessoas com deficiência visual e auditiva em Portugal: Diagnóstico e contributos para uma efetiva inclusão. Lisboa: Universidade Aberta. http://www.isolearn.net/isolearndocs/isolearn_portugal_study.pdf

Rodrigues, F. D. F. (2015). Universidade inclusiva e o aluno com necessidades especiais - $A$ investigação realizada em Portugal (Dissertação de Mestrado). Universidade da Madeira, Funchal.

Runswick-Cole, K. (2011). Time to end bias towards inclusive education?. British Journal of Special Education, 38(3), 112-119. doi:. 10.1111/j.1467-8578.2011.00514.x

Sachs, D., \& Schreuer, N. (2011). Inclusion of students with disabilities in higher education: Performance and participation in student's experiences. Disability Studies Quarterly, 31(2), 99-106. doi: 10.1111/j.1467-8578.2011.00514.x

Santos, E., Gonçalves, M., Ramos, I., Castro, L., \& Lomeo, R. (2015). Inclusão no Ensino Superior: Perceções dos estudantes com NEE sobre o ingresso à universidade. Revista Portuguesa de Educação, 28(2), 251-270. doi: https://doi.org/10.21814/rpe.7741

UNESCO (2017). A guide for ensuring inclusion and equity in education. The Global Education 2030 Agenda. Geneva: UNESCO IBE. Disponível em: http://unesdoc.unesco.org/images/0024/002482/248254e.pdf

United Nations (2015). Convention on the rights of persons with disabilities. Improvement of disability data and statistics: Objectives and challenges. New York: United Nations. Disponível em: http://www.un.org/en/ga/search/ view_doc.asp?symbol=CRPD/CSP/2015/3

Venville, A., Street, A., \& Fossey, E. (2014). Good intentions: Teaching and specialist support staff perspectives of student disclosure of mental health issues in postsecondary education. International Journal of Inclusive Education, 18(11), 11721188. doi:10.1080/13603116.2014.88156

Wessel, R. D., Jones, J. A., Markle, L., \& Westfall, C. (2009). Retention and graduation of students with disabilities: Facilitating student success. Journal of Postsecondary Education and Disability, 117(3), 116-125. Disponível em: http://files.eric.ed.gov/fulltext/EJ831430.pdf 


\section{Legislação}

Lei n. ${ }^{\circ}$ 38/2004. Define as bases gerais do regime jurídico da prevenção, habilitação, reabilitação e participação da pessoa com deficiência. Diário da República - I Série-A, N. ${ }^{\circ} 194$, de 18 de agosto. Disponível em: https://dre.pt/web/guest/ pesquisa/-/search/480708/details/maximized

Lei n. ${ }^{\circ}$ 46/86 (Lei de Bases do Sistema Educativo). Alterada pelas Leis n. ${ }^{\circ} 115 / 97$, de 19 de setembro, 49/2005, de 30 de agosto, e 85/2009, de 27 de agosto, e pela Lei . $^{\circ}$ 65/15, de 3 de julho de 2009. Disponível em: http://www.cnedu.pt/pt/ noticias/cne/1039-lei-de-bases-do-sistema-educativo 


\section{INSTITUTIONAL CHALLENGES TO THE INCLUSION OF STUDENTS WITH SPECIAL EDUCATIONAL NEEDS IN HIGHER EDUCATION}

\section{Abstract}

Based on assumptions of inclusive education and increasing democratization of higher education, the university has been progressively opening up to various minorities, including students with Special Educational Needs (SEN). There is still little knowledge on the subject of diversity and inclusion among teachers and students, in general. Institutional support services to students with SEN need improvement at various levels, namely in terms of the attitudes of teachers and non-teaching staff. What do these institutional actors think about the inclusion of these students? Do the universities provide appropriate conditions to this minority student group? In order to address this issue, we present the results of a study, at the University of Algarve, that aimed to analyze the concepts and attitudes of teachers and non-teaching staff in view of the inclusion of students with SEN in higher education. It was also intended to understand what challenges these institutional actors point out in a context of inclusion. The results presented, with reference to the testimonies of several institutional actors, reveal that, despite the efforts made by the institution, there are still many areas that need more investment: the architectural barriers, the development of more inclusive attitudes of the academic community, teachinglearning practices, and evaluation to ensure equal and fair treatment.

Keywords

Inclusive Education; Special Educational Needs; Higher Education

DÉFIS INSTITUTIONNELS POUR L'INTÉGRATION DES ÉLÈVES AYANT DES BESOINS ÉDUCATIFS SPÉCIAUX DANS L'ENSEIGNEMENT SUPÉRIEUR

Résumé

Fondées sur des hypothèses d'éducation inclusive et démocratisation croissante de l'enseignement supérieur nous avons pu remarquer l'ouverture 
progressive de I'Université à diverses minorités, y compris les étudiants handicapés et les étudiants à besoins éducatifs particuliers. Il est à noter qu'il y a encore peu de connaissances sur le thème de la diversité et l'inclusion parmi les enseignants et étudiants en général, les services de soutien institutionnel aux besoins éducatifs spéciaux manquant d'efficacité à différents niveaux, en particulier au niveau des attitudes des enseignants et du personnel non enseignant. Que pensent les acteurs institutionnels de l'inclusion de ces étudiants? Les universités réunissent-elles les conditions appropriées à cette minorité d'étudiants? Sont présentés les résultats d'une étude de cas à l'Université de l'Algarve visant à examiner les concessions et les attitudes des enseignants et du personnel non enseignant face à l'intégration des élèves dans l'enseignement supérieur et à déceler les difficultés rencontrées par ces acteurs. Les résultats présentent, à travers leurs témoignages, nous constatons que, malgré les efforts déployés par l'institution, il existe encore de nombreux domaines qui ont besoin de plus d'investissements: des barrières architecturales au développement des attitudes plus ouvertes de la communauté universitaire, en passant par des pratiques d'enseignement-apprentissage et d'évaluation qui assureraient un traitement égal et équitable.

Mots-clé

Éducation Inclusive ; Besoins Éducatifs Spéciaux ; Enseignement Supérieur

Recebido em janeiro 2017 Aceite para publicação em outubro 2017

i Departamento de Ciências Sociais e da Educação, Escola Superior de Educação e Comunicação, Universidade do Algarve, Portugal.

ii Departamento de Psicologia e de Ciências da Educação, Faculdade de Ciências Humanas e Sociais, Universidade do Algarve, Portugal.

iii Departamento de Teoría y Historia de la Educación y Pedagogía Social, Facultad de Ciencias de la Educación, Universidad de Sevilla y Grupo de Investigación Estudios Sociales e Intervención Social, España.

iv Escola Superior de Educação e Comunicação, Universidade do Algarve, Portugal.

Toda a correspondência relativa a este artigo deve ser enviada para: Maria Leonor Borges, Universidade do Algarve, Escola Superior de Educação e Comunicação, Universidade do Algarve, Campus da Penha, 8000 Faro. Email: mlborges@ualg.pt 